\title{
Epidemiologia, ciências sociais e políticas de saúde no enfrentamento da COVID-19
}

A pandemia pela COVID-19 pegou o mundo de surpresa. Até o dia 28/04/2020 havia mais de 3 milhões de casos notificados e mais de 210 mil óbitos ${ }^{1}$. Por se tratar de uma doença nova, para a qual a humanidade não possui imunidade, e por ter taxas de transmissão muito elevadas, essa doença pode saturar os serviços de saúde se medidas de distanciamento social não forem adotadas².

Neste fascículo, a Revista Ciência \& Saúde Coletiva publica um temático sobre a COVID-19, fruto da demanda espontânea, no qual são publicados estudos que mostram a contribuição da epidemiologia, das ciências sociais e das políticas de saúde no enfrentamento dessa doença. Sabe-se que o registro de casos da COVID-19 abrange apenas uma pequena parte dos infectados e não se sabe qual a proporção de casos assintomáticos. Hallal et al., por meio de testes rápidos para diagnóstico da COVID-19, a partir da detecção da prevalência da infecção em sintomáticos e assintomáticos, planejam estimar o percentual de casos assintomáticos na população. Isso é muito importante, pois, se o percentual de casos assintomáticos for elevado, a letalidade da doença será mais baixa do que o valor atualmente estimado de $1,4 \%{ }^{3}$. Publicamos o protocolo desse importante estudo, ainda em andamento.

Pedrosa et al. demonstraram que a COVID-19 está migrando para áreas no interior do Ceará com menor distribuição de leitos de terapia intensiva, sendo necessárias medidas de contingência para ampliar o número de leitos de UTI nesses locais. Em outro artigo, Hallal apresentou estatísticas de mortalidade por COVID-19 no mundo, ajustando para o tamanho da população e para o estágio da pandemia em cada país. No mesmo estágio da epidemia, no $30^{\circ}$ dia após o décimo óbito, os países asiáticos apresentaram taxas de mortalidade muito menores do que aquelas observadas nos países europeus.

Em outro artigo, Aquino et al. concluíram que o distanciamento social adotado pela população é efetivo, especialmente quando combinado ao isolamento de casos e à quarentena dos contatos. Bezerra et al., em uma amostra de conveniência de 16.440 usuários de redes sociais, demonstraram que o isolamento social impacta o convívio social, aumenta o estresse, reduz o sono e provoca problemas financeiros para as pessoas de menor renda e escolaridade. Em outro artigo, realizado na Argentina, Johnson et al. concluíram que a população sente medo e angústia, mas também tem responsabilidade e cuidado frente à COVID-19. Castro-de-Araújo et al. revelaram o estresse causado pelo risco eminente de infecção ou incerteza econômica, especialmente em ambientes de baixa e média renda, o que tende a provocar impactos na saúde mental.

Souza analisou a pandemia da COVID-19 no bojo da dinâmica do capitalismo contemporâneo e da globalização, no qual a pandemia (epidemia globalizada) é vista como fruto do capitalismo globalizado. Dentre outras questões, abordaram a priorização dada à esfera econômica, a fragilização dos serviços de saúde, a disseminação de informações falsas na mídia e o maior impacto da epidemia nos grupos mais pauperizados. Deslandes \& Coutinho discutiram as implicações do isolamento social devido à pandemia da Covid-19 para o uso intensivo da internet entre crianças e adolescentes e suas possíveis consequências para a prática de violências autoinflingidas. E, finalmente, Harzheim et al. destacaram o importante papel da atenção primária no Sistema Único de Saúde no Brasil para o enfrentamento da epidemia pela COVID-19.

Antônio Augusto Moura da Silva (https://orcid.org/0000-0003-4968-5138) ${ }^{1}$

Maria Cecília de Souza Minayo (https://orcid.org/0000-0001-6187-9301)²

Romeu Gomes (https://orcid.org/0000-0003-3100-8091) ${ }^{3}$

${ }^{1}$ Departamento de Saúde Pública, Universidade Federal do Maranhão. São Luís MA Brasil.

${ }^{2}$ Escola Nacional de Saúde Pública Sérgio Arouca, Fiocruz. Rio de Janeiro RJ Brasil.

${ }^{3}$ Instituto Fernandes Figueira, Fiocruz. Rio de Janeiro RJ Brasil.

\section{Referências}

1. COVID-19 Dashboard by the Center for Systems Science and Engineering (CSSE) at Johns Hopkins University (JHU). 2020. [acessado 2020 Apr 28]. Disponível em: https://gisanddata.maps.arcgis.com/apps/opsdashboard/index.html\#/bda7594740fd40299423467b48e9ecf6

2. Ferguson NM, Laydon D, Nedjati-Gilani G, Imai N, Ainslie K, Baguelin M, Bhatia S, Boonyasiri A, Cucunubá Z, Cuomo-Dannenburg G, Dighe A, Dorigatti I, Fu H, Gaythorpe K, Green W, Hamlet A, Hinsley W, Okell LC, van Elsland S, Thompson H, Verity R, Volz E, Wang H, Wang Y, Walker PGT, Walters C, Winskill P, Whittaker C, Donnelly CA, Riley S, Ghani AC. Impact of non-pharmaceutical interventions (NPIs) to reduce COVID-19 mortality and healthcare demand. Imperial College London (16-03-2020), doi: https://doi.org/10.25561/77482.

3. Verity R, Okell LC, Dorigatti I, Winskill P, Whittaker C, Imai N, Cuomo-Dannenburg G, Thompson H, Walker PGT, Fu H, Dighe A, Griffin JT, Baguelin M, Bhatia S, Boonyasiri A, Cori A, Cucunubá Z, FitzJohn R, Gaythorpe K, Green W, Hamlet A, Hinsley W, Laydon D, Nedjati-Gilani G, Riley S, van Elsland S, Volz E, Wang H, Wang Y, Xi X, Donnelly CA, Ghani AC, Ferguson NM. Estimates of the severity of COVID-19 disease. Lancet Infect Dis 2020 Mar 30:S1473-3099(20)30243-7. doi: 10.1016/S1473-3099(20)30243-7. Online ahead of print. 